\title{
First results of tests on the WEAVE Fibres
}

\author{
Frédéric Sayède $^{\mathrm{a}}$, Youssef Younes ${ }^{\mathrm{a}}$, Gilles Fasola ${ }^{\mathrm{a}}$, Stéphane Dorent ${ }^{\mathrm{a}}$, Don Carlos Abrams ${ }^{\mathrm{b}}$, \\ J.Alfonso L. Aguerri ${ }^{c}$, Piercarlo Bonifacio ${ }^{a}$, Gavin Dalton ${ }^{\mathrm{dg}}$, Kevin Dee ${ }^{\mathrm{c}}$, Philippe Laporte ${ }^{\mathrm{a}}$, Ian \\ Lewis $^{\mathrm{g}}$, Emilie Lhome ${ }^{\mathrm{b}}$, Kevin Middleton ${ }^{\mathrm{d}}$, Johan H. Pragt ${ }^{\mathrm{e}}$, Juerg Rey ${ }^{\mathrm{b}}$, Remko Stuik ${ }^{\mathrm{e}}$, Scott C. \\ Trager $^{\mathrm{f}}$
}

\author{
${ }^{a}$ GEPI, Observatoire de Paris, Meudon, France, \\ ${ }^{\mathrm{b}}$ Isaac Newton Group of Telescopes, Santa Cruz de La Palma, La Palma, Spain, \\ ${ }^{\mathrm{c}}$ Instituto de Astrofisica de Canarias, La Laguna, Tenerife, Spain, \\ ${ }^{\mathrm{d}}$ Rutherford Appleton Laboratory RAL Space, Didcot, United Kingdom, \\ ${ }^{\mathrm{e}}$ NOVA Optical Infrared Instrumentation Group, Dwingeloo, Netherlands, \\ ${ }^{\mathrm{f}}$ Kapteyn Astronomical Institute, University of Groningen, Groningen, Netherlands. \\ ${ }^{\mathrm{g}}$ University of Oxford, United Kingdom
}

\begin{abstract}
WEAVE is a new wide-field spectroscopy facility proposed for the prime focus of the $4.2 \mathrm{~m}$ William Herschel Telescope. The facility comprises a new 2-degree field of view prime focus corrector with a 1000-multiplex fibre positioner, a small number of individually deployable integral field units, and a large single integral field unit. The IFUs (Integral Field Units) and the MOS (Multi Object Spectrograph) fibres can be used to feed a dual-beam spectrograph that will provide full coverage of the majority of the visible spectrum in a single exposure at a spectral resolution of $\sim 5000$ or modest wavelength coverage in both arms at a resolution $~ 20000$. The instrument is expected to be on-sky by 2017 to provide spectroscopic sampling of the fainter end of the Gaia astrometric catalogue, chemical labeling of stars to V 17, and dedicated follow up of substantial numbers of sources from the medium deep LOFAR surveys.

After a brief description of the Fibre System, we describe the fibre test bench, its calibration, and some test results. We have to verify 1920 fibres from the MOS bundles and 740 fibres from the mini-IFU bundles with the test bench. In particular, we present the Focal Ratio Degradation of a cable.
\end{abstract}

Keywords: WEAVE, Optical fibres, Test plan, Multi Object Spectroscopy, WHT, MOS.

\section{INTRODUCTION}

WEAVE [1,2] is a new wide-field spectroscopy facility proposed for the prime focus of the $4.2 \mathrm{~m}$ William Herschel Telescope. The facility comprises a new 2 degree field of view prime focus corrector [4,7] with a 1000-multiplex fibre positioner [5,8], a small number of individually deployable integral field units, and a large single integral field unit. The IFUs and the MOS fibres can be used to feed a dual-beam spectrograph [6] that will provide full coverage of the majority of the visible spectrum in a single exposure at a spectral resolution of $\sim 5000$ or modest wavelength coverage in both arms at a resolution $\sim 20000$. The instrument is expected to be on-sky by 2017 to provide spectroscopic sampling of the fainter end of the Gaia astrometric catalogue, chemical labelling of stars to V 17, and dedicated follow up of substantial numbers of sources from the medium deep LOFAR surveys.

The WEAVE fibres ensure the link between the positioner and the spectrograph (figure 1). WEAVE will be installed at the prime focus. There are about 960 single fibres on each focal plate and twenty mini-IFUs on one of these plates. We manufactured a prototype of a MOS bundle and will begin to manufacture the $10 \%$ first bundle thanks to the DIM/ACAV budget from the Region Ile-de-France**. To test all the MOS and mini-IFU cables from the prototype to the remaining cables production, we are developing a test bench.

*frederic.sayede@obspm.fr ; phone: (33) 145077650

**www.dim-acav.org 


\section{THE MOS FIBRE SYSTEM}

This mode presents a very high multiplex capacity [1], since it is a network of 960+960 single fibres. 20 of them are mounted but will be used only if there are broken fibres. Each single fibre presents a 1.3" circular input aperture on the sky. The two plates of the positioner are equipped with the MOS mode: MOS A (40 bundles) and MOS B (40 bundles) for respectively the first and the second focal plate.

The output aperture of a fibre fits the input aperture with some degradation. The aperture of the spectrograph is F/3.1. As the aperture of the telescope is F/2.75, a diverging lens slows down the beam into F/3.2 equivalent in air (Figure 1). The sky aperture of 1.3 " is defined by the fibre core diameter and the scale given by the "telescope + corrector" focal.

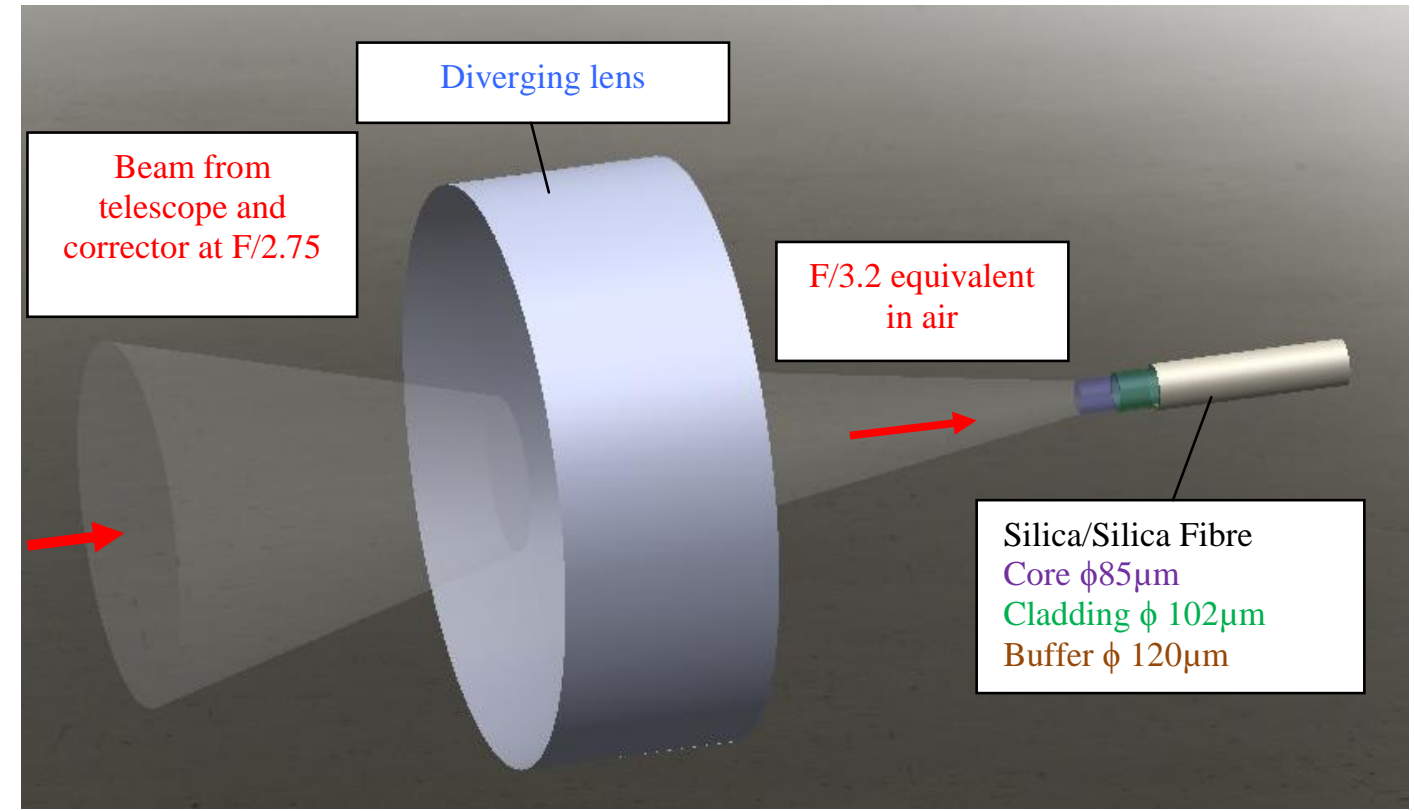

Figure 1: Fibre injection principe (focus injection)

The MOS bundles include:

- 80 cables of 24 optical fibres and the associated sheaths

- 1920 buttons

- 80 slitlets

- spare of different components (buttons, slitlets...)

MOLEX (formerly Polymicro Technologies) manufactures the optical fibre used for the MOS subsystem. The type of the fibre is the FBP 085/102/120 [3]. The Figure 2 gives the characteristics of the fibre.

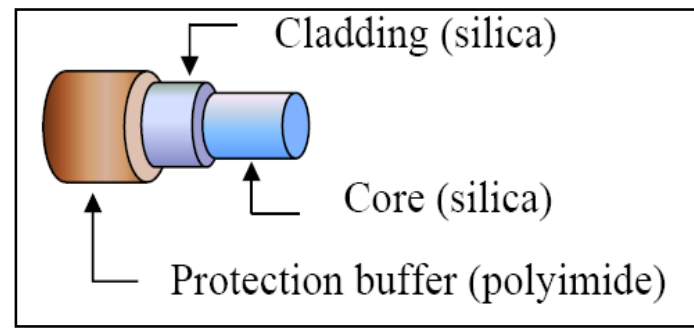

\begin{tabular}{|l|c|}
\hline \multicolumn{1}{|c|}{ Type } & Value \\
\hline Core diameter & $85 \mu \mathrm{m}+/-3 \mu \mathrm{m}$ \\
\hline Cladding diameter & $102+/-3 \mu \mathrm{m}$ \\
\hline Protection buffer diameter & $120+/-3 \mu \mathrm{m}$ \\
\hline Numerical Aperture & $0.22+/-0.02$ \\
\hline${\text { Operating } \mathrm{T}^{\circ}}^{\circ}$ & $-65^{\circ} \mathrm{C} /+300^{\circ} \mathrm{C}$ \\
\hline
\end{tabular}

Figure 2: characteristics of the MOS Fibre

The MOS bundles (Figure 3) are grouped four by four with an External Conduit and gathers $4 \times 24$ single fibres. The SEDI-ATI company (France) manufactures a prototype of the MOS bundle. 


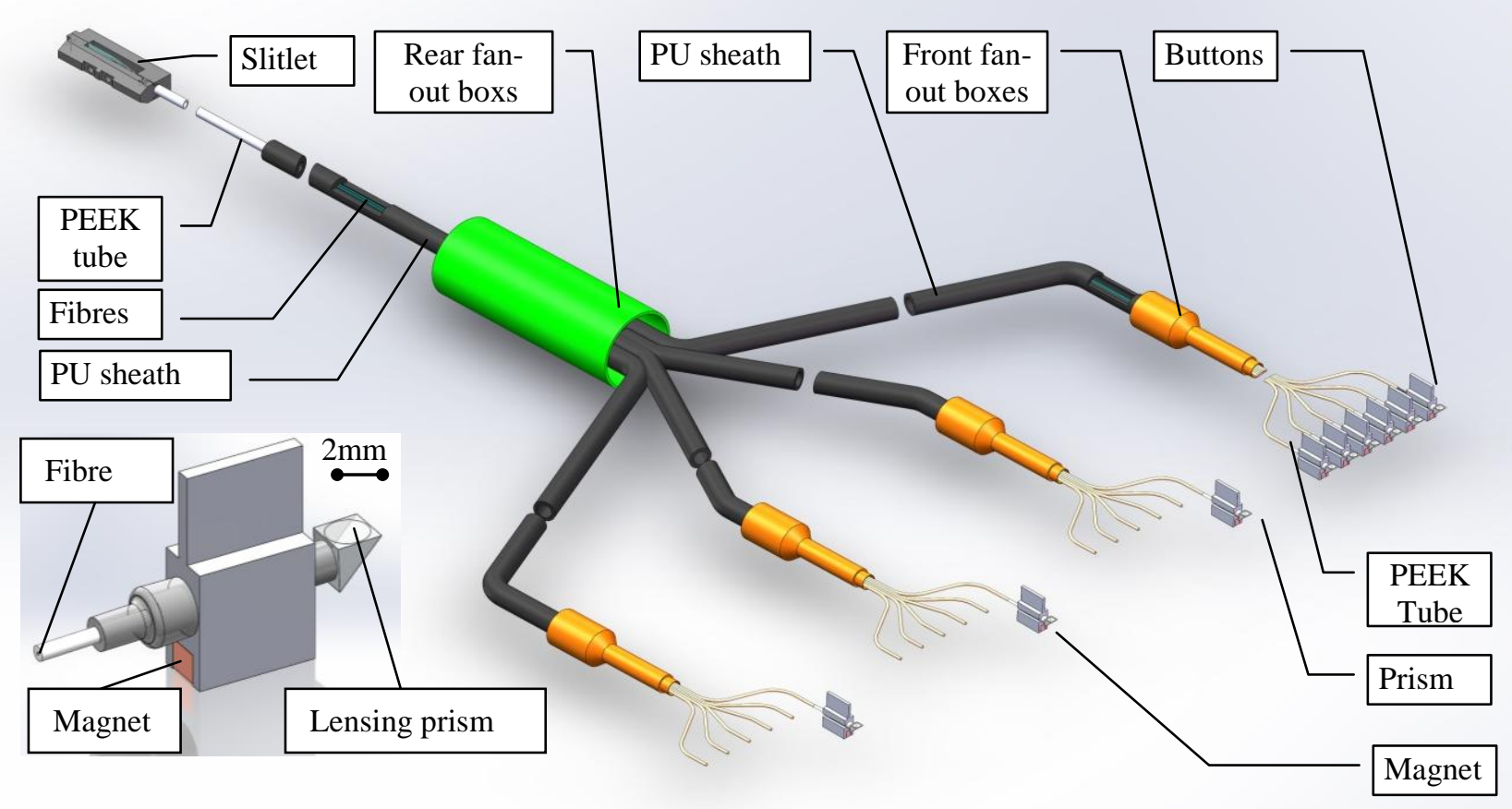

Figure 3: MOS bundle

\section{THE TEST BENCH}

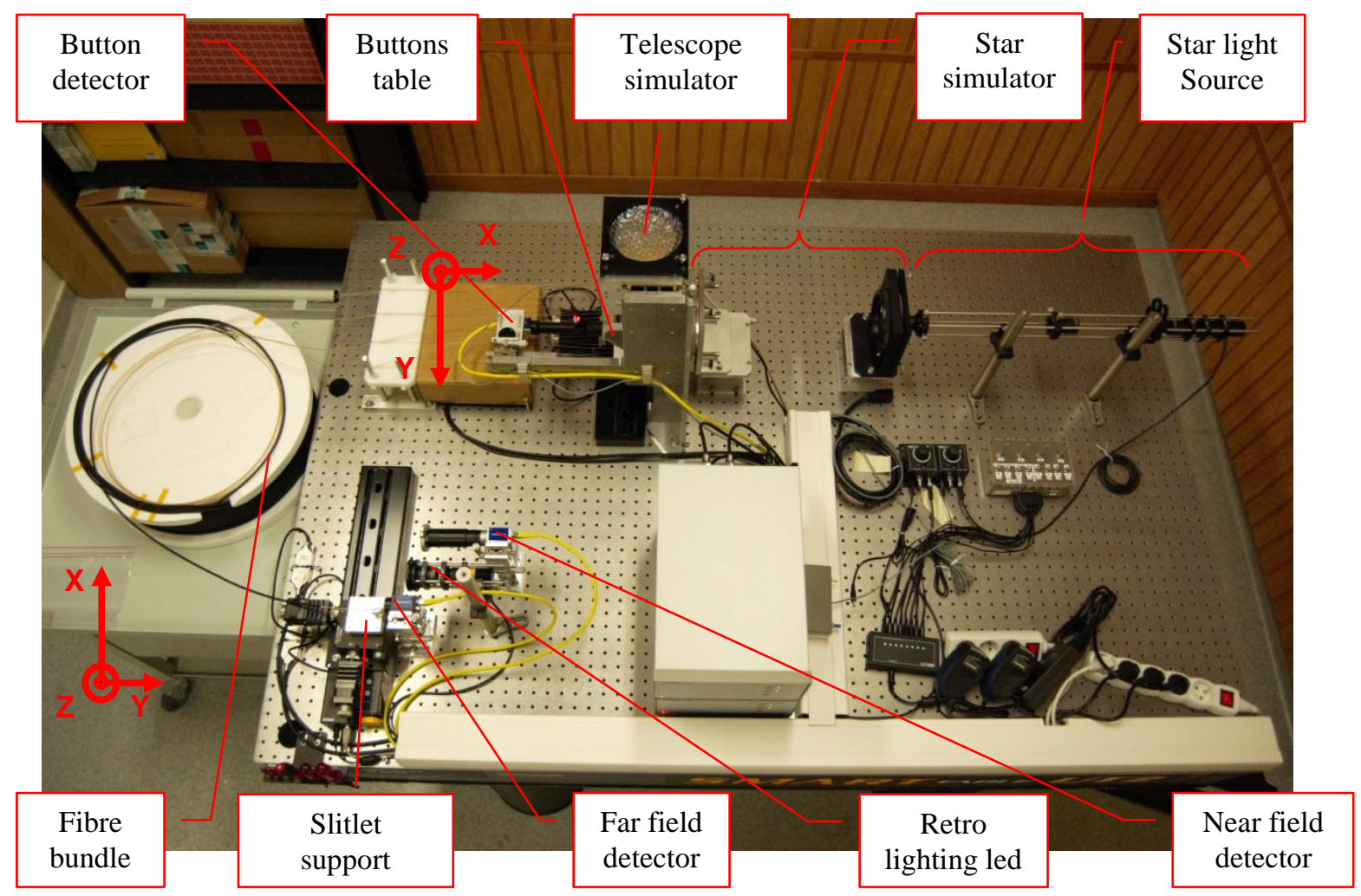

Figure 4: global view of the test bench 
The test bench is used to verify and evaluate the performances of the MOS, the mIFU and the Guide fibre bundles in accord with the Fibre System MAIT Plan [9]. At list it will be automated. We are building the bench in three steps to reduce risk. During the first step we designed and manufactured the source and the analysis parts of the bench. These parts are required for the first bench use. During the second step, we designed and produced the motorized parts used to hold and accurately move the buttons and slitlets in front of the source, the analysis systems, etc. During the third step, we will make the bench totally automatic. The test bench controls and tests the optical and physical characteristics of the entrance and output fibre bundles.

The test bench is composed of:

- The light sources

- The telescope simulator

- The table with the button holders

- The table with the slitlet holders

- The analysis systems

- The control command and the software analysis

- The bench and the environment

\subsection{Sources}

We need three sources:

- a white source (about 370nm to 900nm) (Figure 5)

- a green source (about 530nm)

- a red source (about 650nm)
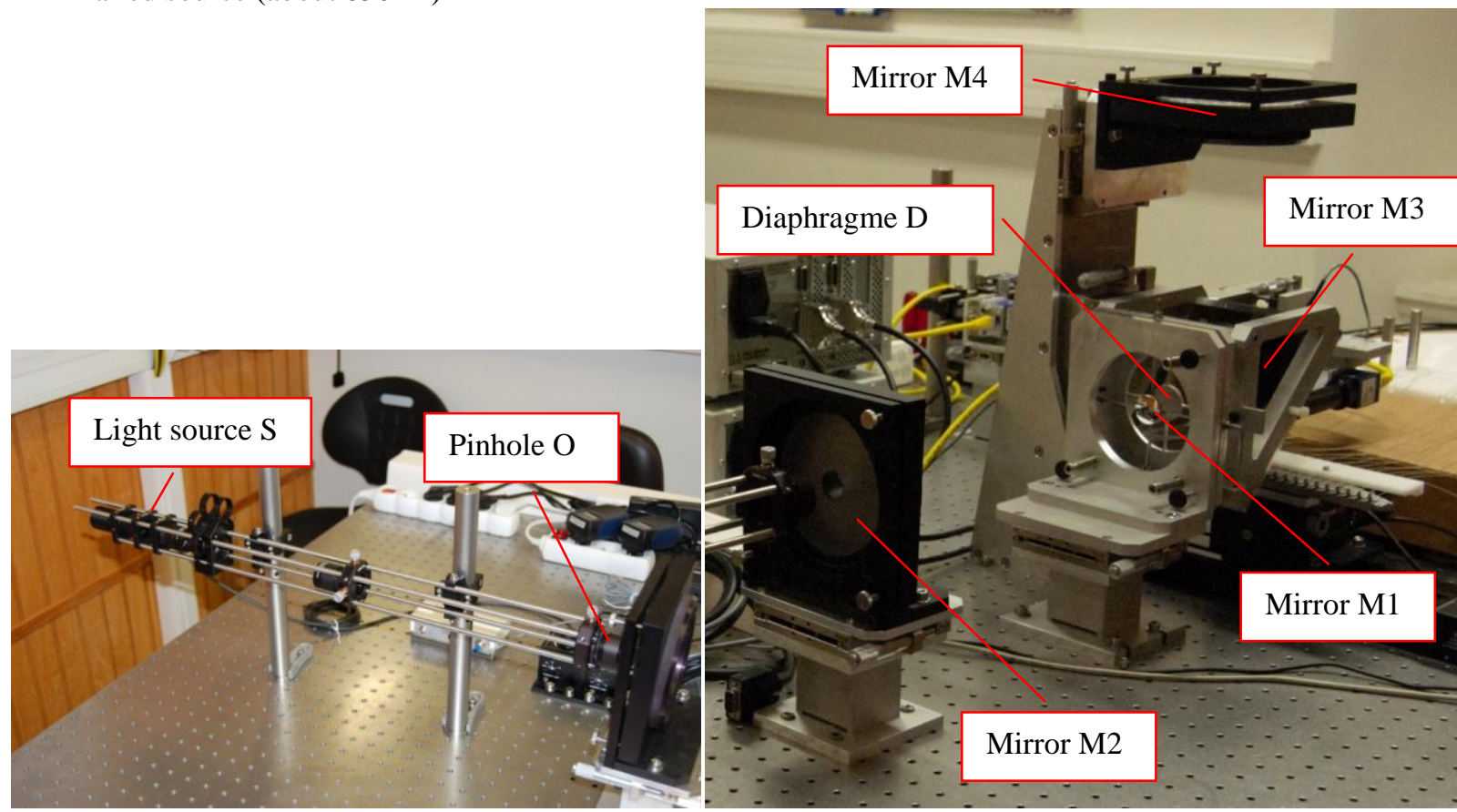

Figure 5: Light source (left), star and telescope simulators (right) of the test bench

\subsection{Telescope simulator}

The main parts of the test bench telescope simulator (figure 5 and 6) are:

- A light source $\mathrm{S}$ with a pinhole $\mathrm{O}$.

- A reverse mode collimator telescope made of M1, M2 and M4 (M0 does not exist on the current bench) mirrors as star or a spatial extended object depending on the object $\mathrm{O}$. 
- The WHT simulator made of a diaphragm (D) as wire and secondary mirror obstruction simulator and the M3 mirror as WHT primary mirror simulator.

- $\quad$ The image focal plane $(\mathrm{F})$.

The requirements of the telescope simulator are given in Table 1:

\begin{tabular}{|c|c|c|c|c|}
\hline Spec & Designation & Value & Tolerances & Remarks \\
\hline RTS 01 & Output aperture & $\mathrm{F} / 2.7$ & \pm 0.2 & \\
\hline RTS 02 & Telecentricity & partial & - & Object side at list \\
\hline RTS 03 & Minimum image size in $(\mathrm{F})$ & $50 \mu \mathrm{m}$ & $\pm 10 \mu \mathrm{m}$ & FWHM (=0.9") \\
\hline RTS 04 & Maximum image size in $(\mathrm{F})$ & $400 \mu \mathrm{m}$ & $\pm 10 \mu \mathrm{m}$ & FWHM (=7’') \\
\hline RTS 05 & $\begin{array}{l}\text { Centre obstruction } \\
\text { Wire width }\end{array}$ & $\begin{array}{l}1 / 2.47 \text { of } \phi \text { pup } \\
1 / 70 \text { of } \phi \text { pup }\end{array}$ & $\begin{array}{l} \pm 5 \% \\
\pm 5 \%\end{array}$ & $\begin{array}{l}\text { Obstruction design as the } \\
\text { WHT }\end{array}$ \\
\hline RTS 06 & Number of wire & 4 & - & $90^{\circ}$ \\
\hline
\end{tabular}

Table 1: requirements of the telescope simulator

The actual optical design (Figure 6) gives for an object $(\mathrm{O})$ diameter from $0.4 \mathrm{~mm}$ to $1.2 \mathrm{~mm}$ an image from $50 \mu \mathrm{m}$ to $150 \mu \mathrm{m}$ (Figure 6). We can extend the object $(\mathrm{O})$ size to increase the image size.



Figure 6: optical design of the test bench

The image quality is less than $20 \mu \mathrm{m}$ PTV on more than the fibre diameter (Figure 7). 


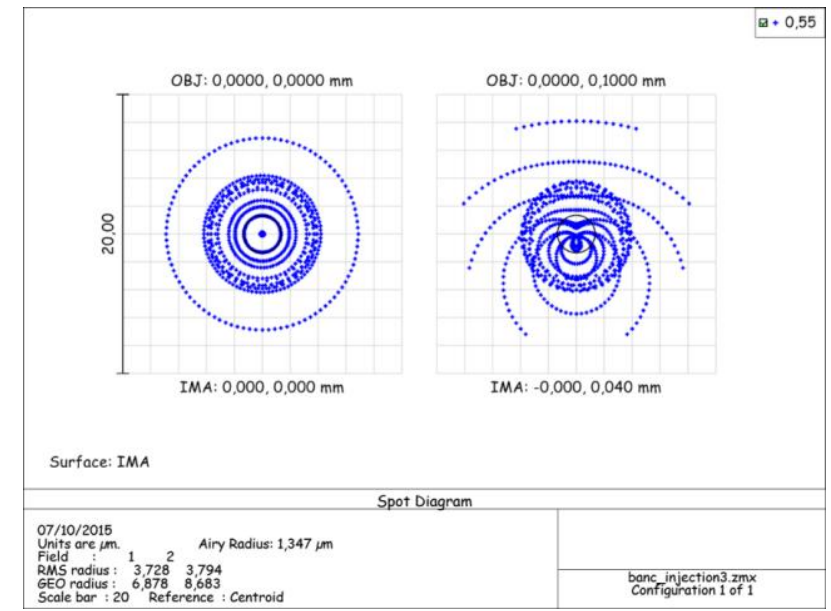

Figure 7: PSF of the bench optics (light source included)

\subsection{The table with the button holders}

The button holders receive the buttons (Figure 8) with an absolute position accuracy equivalent to what the positioner is able to do.

To calibrate this absolute position, we use a reference spot of $75 \mu \mathrm{m}$ imaged on a button camera. We put the hole in correspondence with the source (maximum of luminosity with a $0.6 \mathrm{~mm}$ diameter object source $\mathrm{O}$ ) and translate the table to reach each theoretical position of the button. We then observe the mechanic of each button. The mechanic of the button is correct. It was controlled in a previous step of the project [9], the system is able to calculate the statistical and systematic error in the position of the button. The translation tables are very accurate so as not to disturb the precision of the measurements.
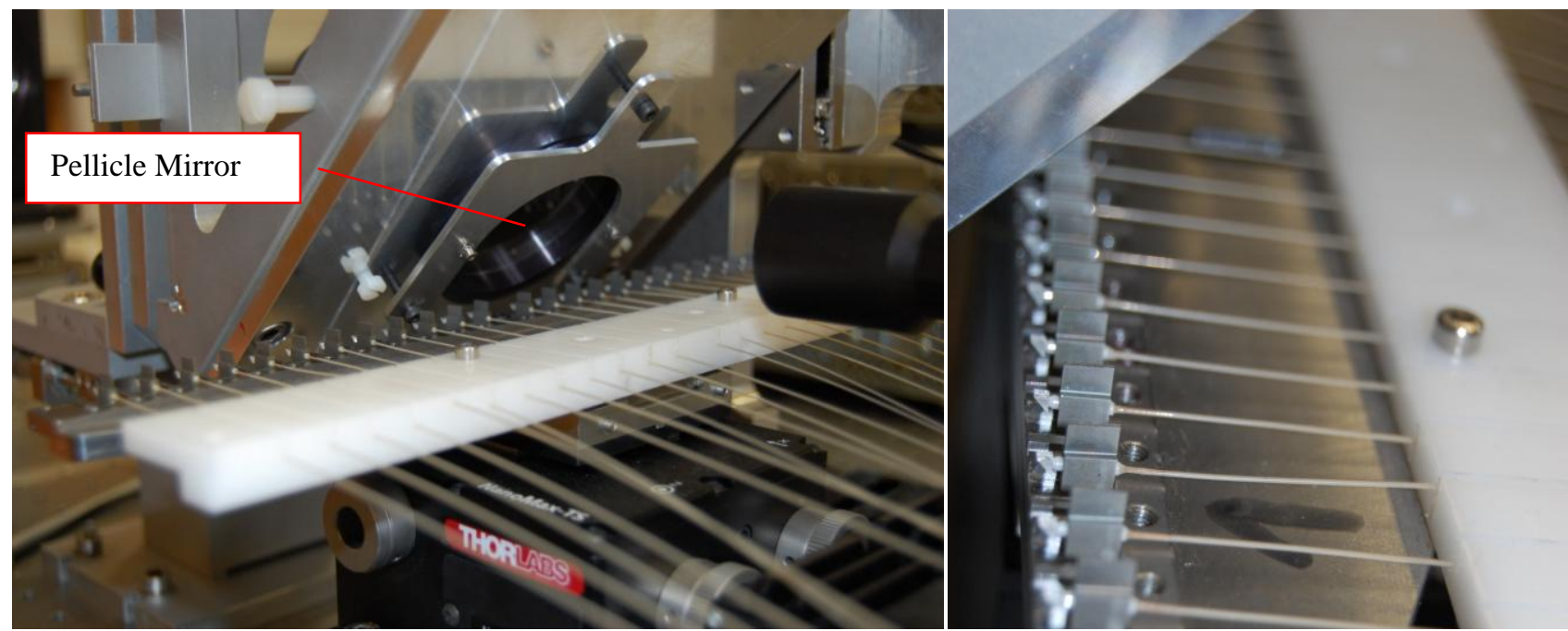

Figure 8: button holders with buttons and fibres

\subsection{The table with the slitlet holder}

The slitlet is mounted on the test bench (Figure 9) as on the spectrograph slit. There is no accuracy for the re-positioning in the $\mathrm{Y}$ direction (optical fibre axis). 


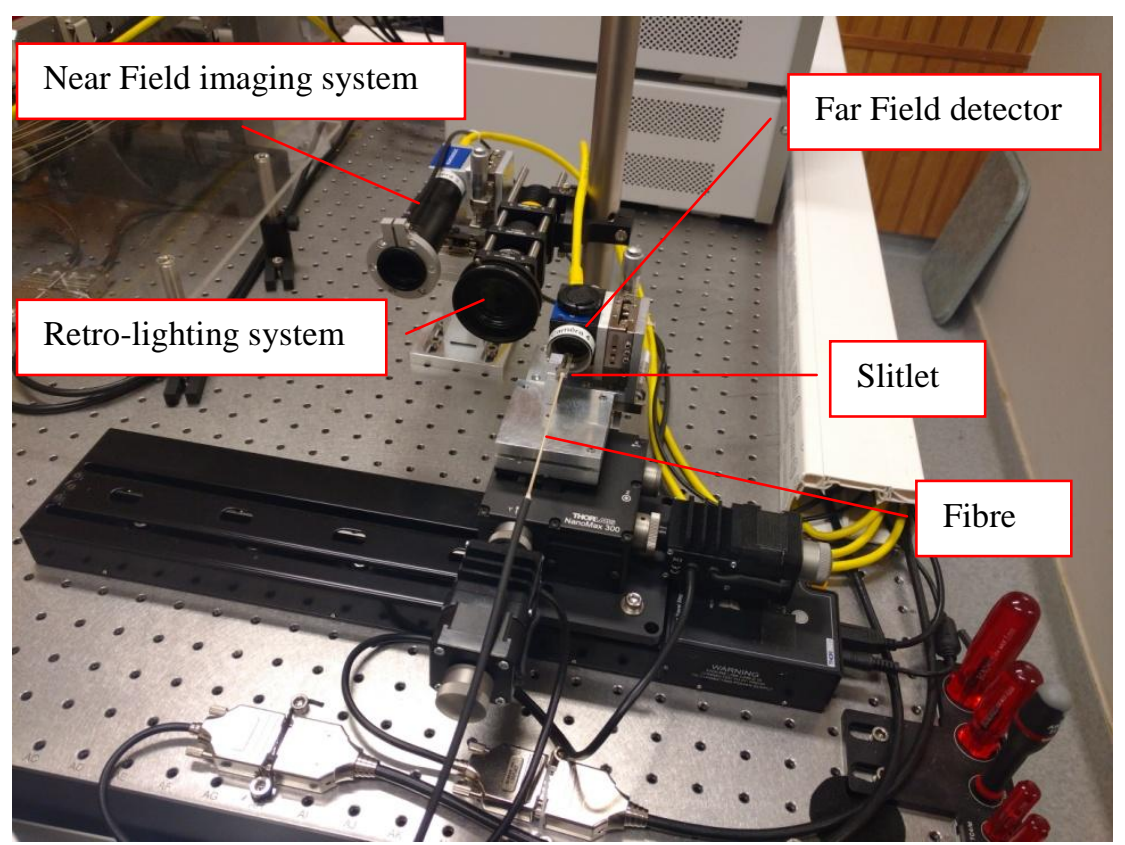

Figure 9: slitlet holder and the translations

\subsection{The analysis systems}

- At the bundle input

A pellicle mirror images the fibre entrance into the button (Figure 10). The object focal plan of the imaging system is the fibre extremity. The imaging system sees simultaneously the projection of the injection light on the button and the back lighted fibre front face (Figure 12). The button positioning system put the fibre core centre onto the light source spot centre. When they are superimposed, we will note the position into the position calibration table. Then we can begin the control/test of the bundle.

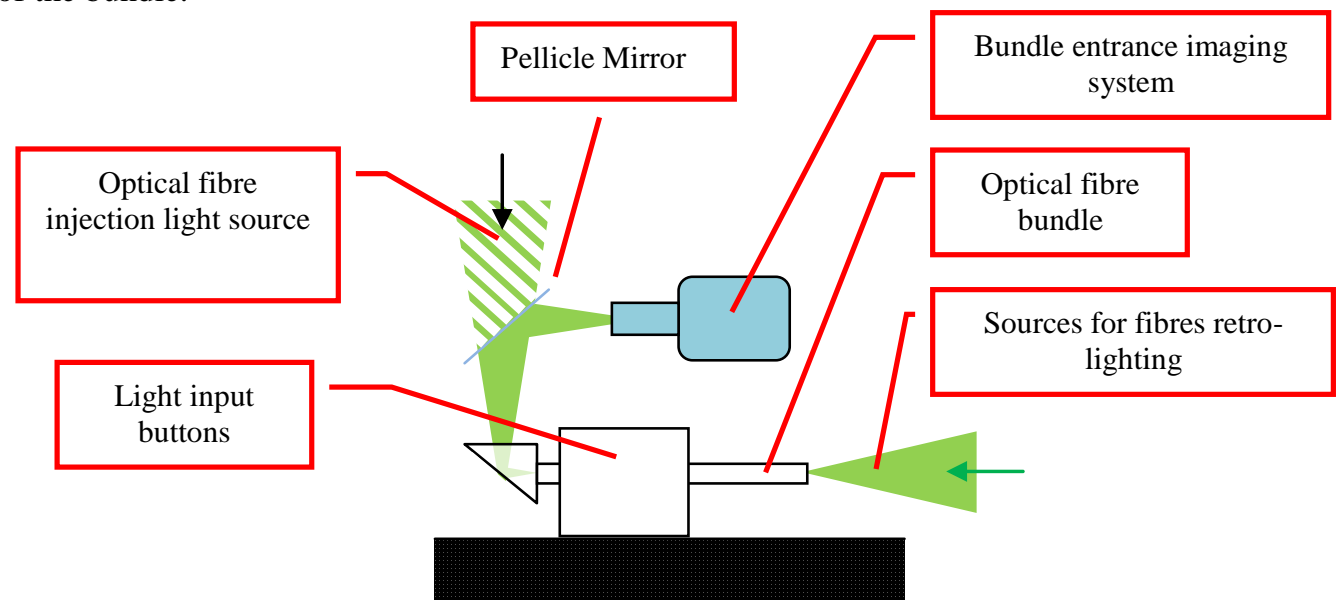

Figure 10: Fibre core and injection spotlight imaging

- At the bundle output

With a similar calibration and a similar method of measurement, we will control the absolute position of each fibre of the slitlet. We will also control or test all the requirements relative to the light transmission. 




Figure 11: global test sequence 


\subsection{The control command and the software analysis}

According the large number of fibre to be characterized and the accuracy needed, a human process cannot be envisaged. We investigated several solutions before purchasing a system based on the DataLogic software to manage all the image processes and the Labview software to control the motors and makes the smart calculations.

The DataLogic software provides high-level tools to study images such as circle recognition, contrast estimates, reference position, etc. We created an algorithm that uses some of these tools to make all the geometric measurements on the fibres (prism, lens, fibre dimensions...).

The automation is performed by the LabView software, which initializes the bench electronics and tables and proceeds through each step of the measurement process as described below. For each step, it saves the image acquired by our DataLogic algorithm for archive purpose and proceeds to move the fibre on the bench while saving the data on the geometry estimated by our algorithm.

\section{SOME TEST ON THE PROTOTYPE}

\section{- The fibre diameter verification}

This is measured in the sequence 8 of the global test sequence (Figure 11).The star light illumination permits the measurement of the fibre diameter. The size is automatically detected in the automatic mode at the output of the fibre. This measurement has been done manually for the prototype bundle.

We intend to have a continuous feed in fibres, but the fibre diameter could change if the company doesn't take care. The measurement is systematic for each fibre with the automatic process.

The Datalogic vision software is able to find enough points on the core diameter to determine the size (Figure 12).

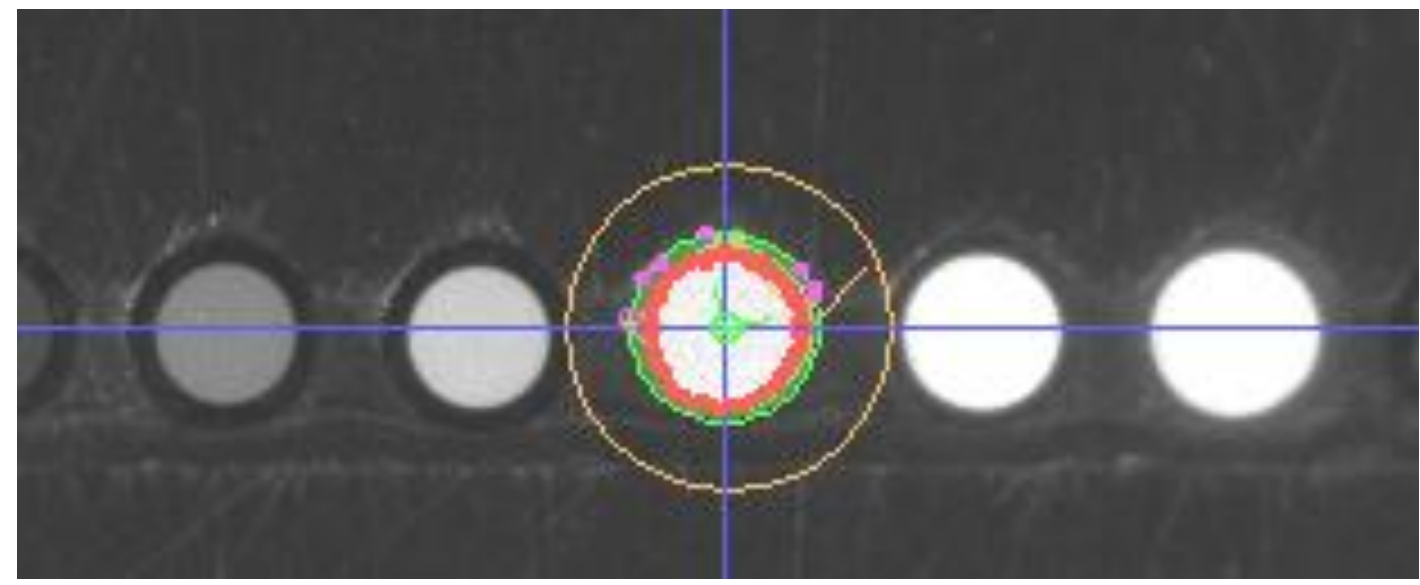

Figure 12: automatic measure of the fibre core diameter and position

- The position of the entrance fibre core centre versus the mechanics

This is measured in the sequence 7 of the global test sequence (Figure 11). All the buttons are mechanically at a fixed position on the test table. The motorized translations are able to determine precisely the theoretical position of each mechanical button (after a global calibration using always the same button). For each button we have to verify (and to measure) the position of the image fibre centre versus the button mechanics. Due to two different $Z$ positions of the fibre image and the mechanic, we need to focus with a $\mathrm{Z}$ displacement during the measure. The quality of the translation and of the mechanics ensures a displacement without shifting. The telecentricity of the optical objective ensures also an accuracy of one pixel for each measure.

The process is the following (DS mean Datalogic System and LS is the Labview Software): 

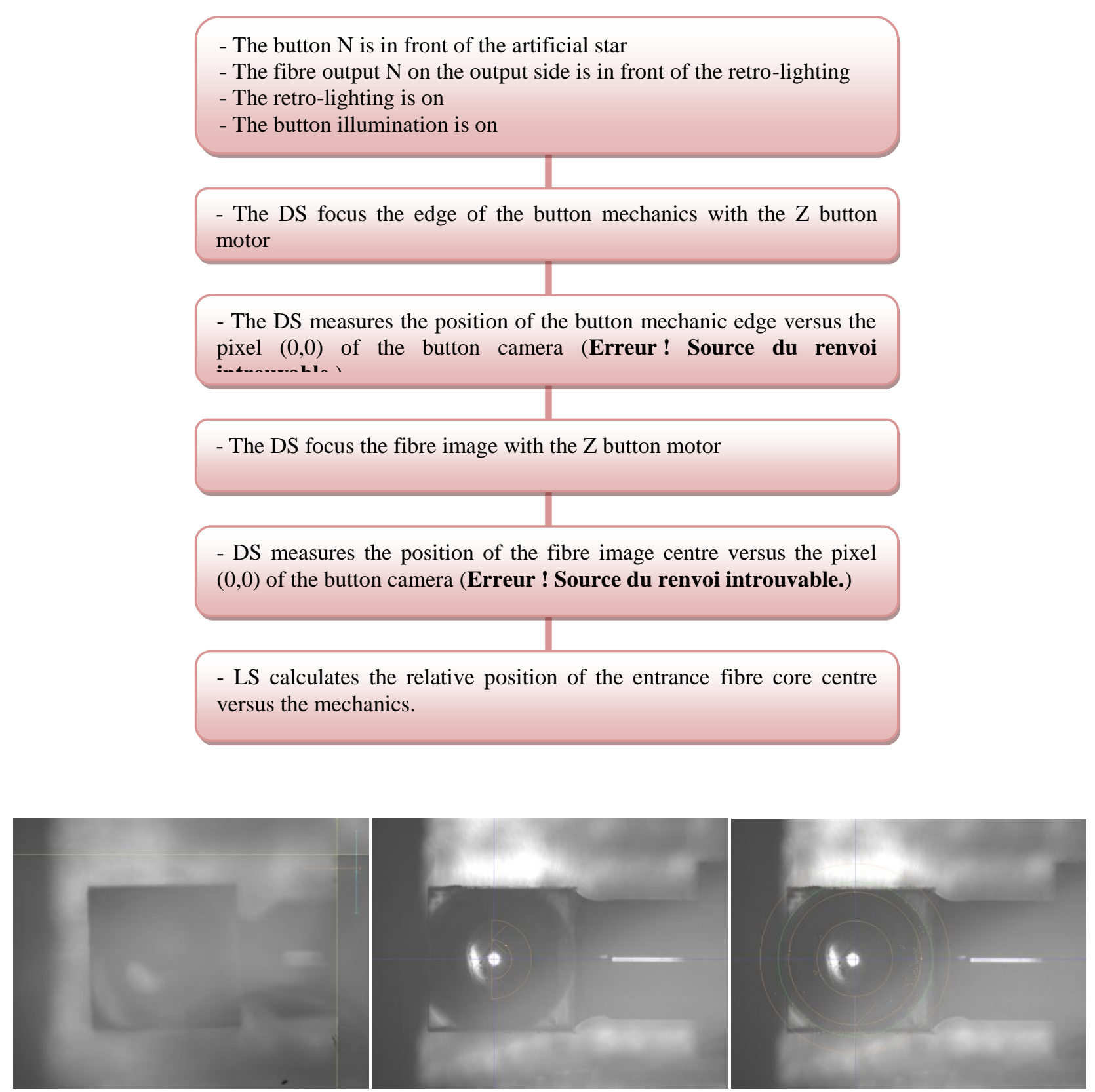

Figure 13: measurements of the position of the button mechanic edge versus the pixel $(0,0)($ left $)$, measurements of the position of the fibre image centre versus the pixel $(0,0)$ (centre), determination of the lens centre of the prism.

\section{- Measuring the F/D at the output of the fibre}

In order to measure the F/D ratio at the exit of the fibre we used the following procedure.

1. We place the camera as close as possible to the slitlet and take a bias frame.

2. we illuminate the fibre from the button side, through the telescope simulator and take another image

3. we move the camera away from the slitlet by a know value (ie $4 . \mathrm{mm}$ ) and take another bias frame with no light on the fibre

4. we illuminate the fibre and take another image

The result of the two images when the fibre is illuminated is shown in Figure 14. 



Figure 14: Two images taken with the fibre illuminated from the button end, through the telescope simulator. The image in the left panel is taken close to the slitlet, while the image is taken at a distance of $\mathbf{4} \mathbf{m m}$ from the first one

We wrote a simple IDL procedure to process these four images. First the bias taken at the same position is subtracted from each image. A small amount of ambient light is still present after this subtraction, at an average level of about 1 count. The procedure measures this background in 37 positions, centered at 40 pixels from the edge of the detector, by taking the average value over a square window of 11 pixels side. The background across the entire detector is then estimated by fitting a plane to these points, through chi-squared minimization (IDL function "sfit"). The fitted background is then subtracted. A two dimensional Gaussian is then fitted to the resulting image using the procedure "mpfit2dpeak" [10].

The result of the fitted Gaussian surface is shown as a surface plot in Figure 15, right panel, while the original image is shown in the left panel.
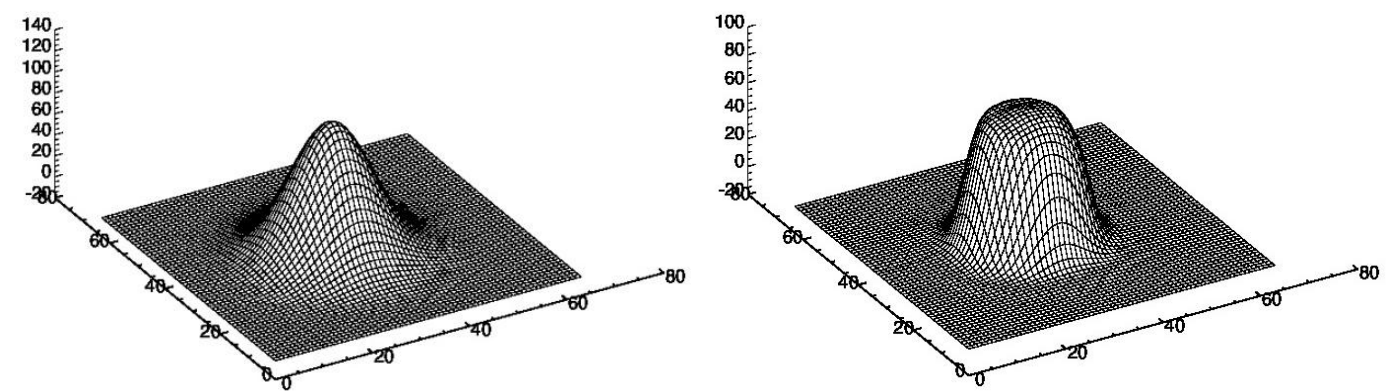

Figure 15: A surface plot of the image taken at the exit of the slit (left panel) and the surface of the corresponding Gaussian fit (right panel)
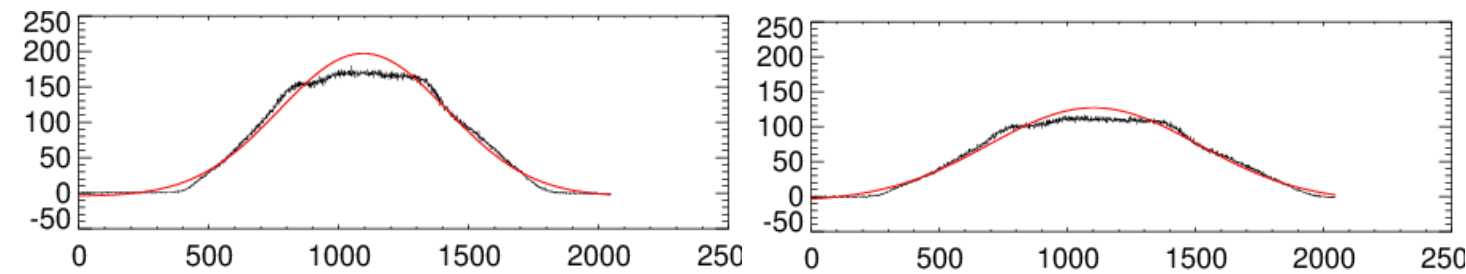

Figure 16: Cross-cut along the $x$-axis in correspondence to the $y$ of the centre of the Gaussian fit of the image at the exit of the slit (top panel) and $4 \mathrm{~mm}$ away (bottom panel). The red line is the corresponding cross-cut on the Gaussian fit. 
A cross-cut along the central y column of the two images is shown in Figure 16, along with the corresponding cross-cut of the best fitting Gaussian surface. While it is clear that the images are not perfectly described by a Gaussian shape, since they are too flat-top, it is also apparent that the Gaussian fit provides a robust estimate of the width of the image. To estimate the F/D we take as width of each image the average of the full widths at half maximum of the Gaussian in $x$ and $y$. The two differ by less than 10 pixels, since the images are fairly symmetric. We transform these into lengths in $\mathrm{mm}$, by using the pixel size $(9 . \mathrm{e}-3 \mathrm{~mm}$ ) and use the formula on the next paragraph, using $\mathrm{p}=4 \mathrm{~mm}$ to compute the F/D.

We then integrate the whole image in squares of different size, centred on the centre of the Gaussian, and normalize these integrals to the integral over the whole detector. This ratio is considered an estimate of the energy fraction included in any given aperture "d". We assign the value F/D derived above to the integral over a width corresponding to the FWHM of the Gaussian, let us call it $(\mathrm{F} / \mathrm{D})_{0}$. We then assign the fraction of energy ensquared in any given aperture to the ratio:

$$
\mathrm{F} / \mathrm{D}=(\mathrm{F} / \mathrm{D})_{0} \times\left(\frac{\mathrm{FWHM}}{d}\right)
$$

\section{- Measure of the FRD losses}

To automate the process using the Datalogic Vision Software (DVS), we first use the above method to determine the distance $s$ of the Figure 17. Using the two pictures we find the s distance with a very good accuracy:

$$
\mathrm{s}\left(1^{\mathrm{st}} \text { picture }\right)=14.826 \mathrm{~mm} \text { and } \mathrm{s}\left(2^{\mathrm{nd}} \text { picture }\right)=14.851 \mathrm{~mm} \text { with } \delta \mathrm{s}=25 \mu \mathrm{m}=>\mathrm{s}=14.839 \mathrm{~mm} \pm 13 \mu \mathrm{m}
$$

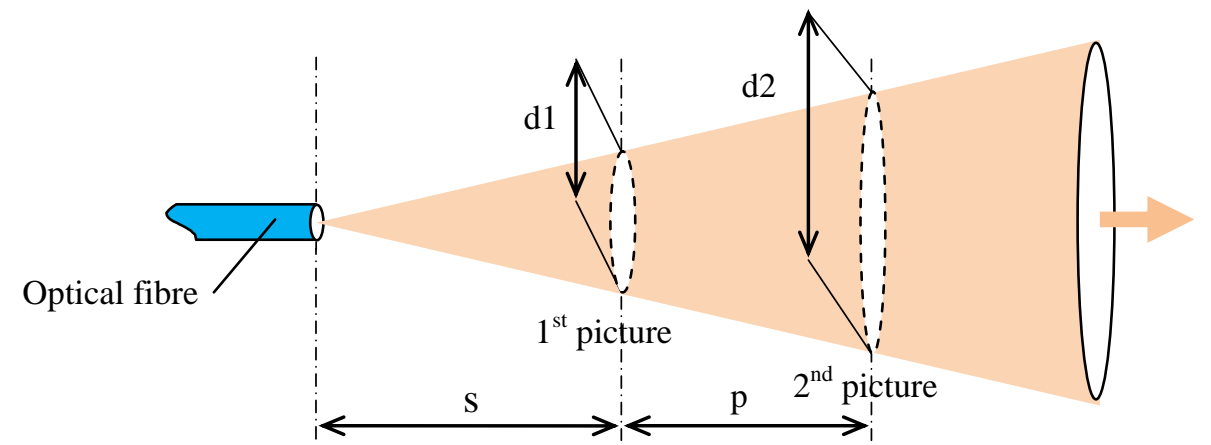

Figure 17: output beam - Measure of the Far Field

We calculate the scale on the detector: $\mathrm{F} / \mathrm{N}$ as a function of pixel numbers. $\mathrm{F} / 3$ is a circle of 550pixels on the nearest image.

With the DVS, we evaluate the noise at different edges of the signal pictures (Figure 14), the total signal $\mathrm{E}_{\text {total }}$ and the signal $\mathrm{E}_{\text {signal }}$ into an $\mathrm{F} / \mathrm{N}$ circle. We calculate the total surface and the signal surface into the $\mathrm{F} / \mathrm{N}$ circle. We deduce $\mathrm{B}_{\text {total }}$ and $\mathrm{B}_{\text {signal }}$, the total noise and the signal noise. The percent of energy $\% \mathrm{E}$ passing through an $\mathrm{F} / \mathrm{N}$ aperture is given by:

$$
\% E=\frac{E_{\text {signal }}-B_{\text {signal }}}{E_{\text {total }}-B_{\text {total }}}
$$

By varying $\mathrm{N}$ we construct a plot of \%Energy as a function of F/D (figure $\mathrm{N}+2$ ).

The aperture is given by:

$$
\frac{F}{D}=\frac{p}{d 2-d 1}
$$

The error on the F/D calculation is:

$$
\partial\left(\frac{\mathrm{F}}{\mathrm{D}}\right)=\frac{\partial p}{d 2-d 1}+\frac{\mathrm{p} \times \partial(\mathrm{d} 2-\mathrm{d} 1)}{(d 2-d 1)^{2}}
$$


The translator accuracy between two position is less than $\pm 2 \mu \mathrm{m}=>\delta \mathrm{p}=0.004 \mathrm{~mm}$

The pixel detector is about $9 \mu \mathrm{m}$ and the diameter evaluation process is stable at \pm 10 pixels $=>\delta(\mathrm{d} 2-\mathrm{d} 1)=0.09 \mathrm{~mm}$.

We can conclude of the losses by FRD for this MOS fibre of the prototype bundle for a F/3.1 spectrograph: about $11 \% \pm 1.5 \%$.

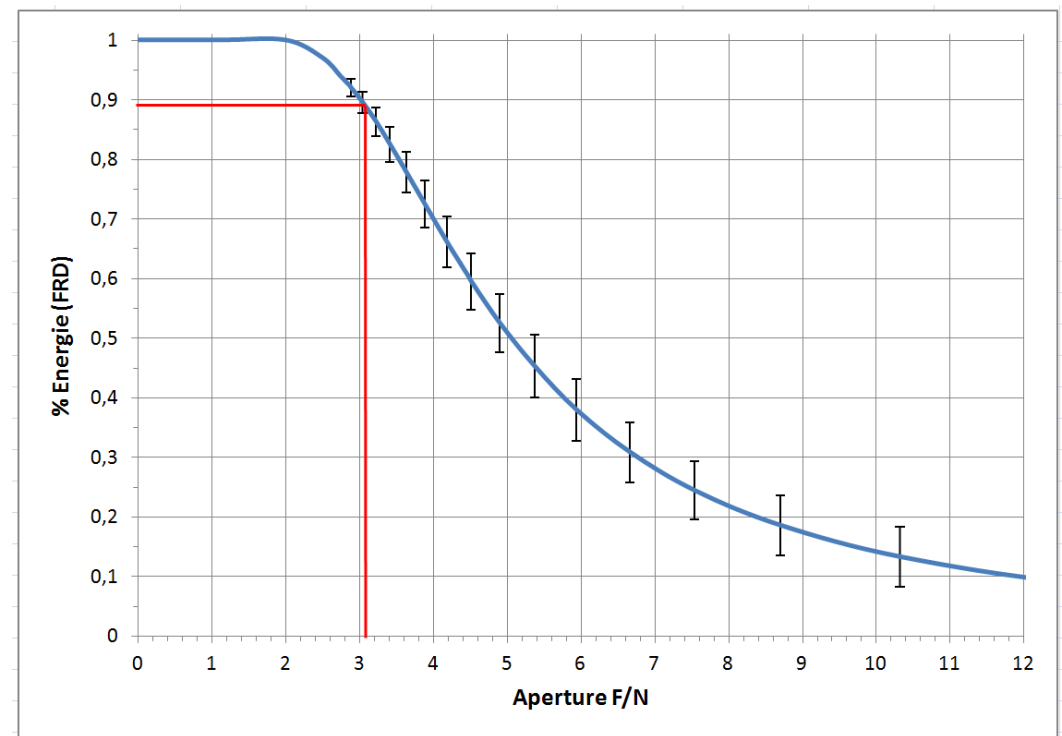

Figure 18: losses by FRD: percent of Energy function of the aperture F/N

\section{CONCLUSION}

During this phase, we are tested and controlled the MOS prototype using the test bench. We have to automate it to control and test the future 4000 fibres manufactured by the industry, before to send them for integration to the William Herschel Telescope.

\section{REFERENCES}

[1] Dalton G. et al, "WEAVE: the next generation fibre spectroscopy facility for the William Herschel telescope: project overview and update on the final design stage", Proc. SPIE 9147-20 (2014)

[2] Dalton G. et al, "WEAVE: the next generation fibre spectroscopy facility for the William Herschel telescope", Proc. SPIE 8446-23 (2012)

[3] Guinouard I., Bonifacio P., Trager S. C., Verheijen M. A. W., Lewis I., Dalton G., "Development of the single fibres and IFUs of WEAVE", Proc. SPIE 8450-127 (2012)

[4] Abrams D. C. et al, "The mechanical design for the WEAVE prime focus corrector system", Proc. SPIE 9147-93 (2014)

[5] Lewis I. J. et al, "The fibre positioning system concept for WEAVE at the William Herschel telescope", Proc. SPIE 9147-117 (2014)

[6] Rogers K. et al, "The design of the WEAVE spectrograph", Proc. SPIE 9147-242 (2014)

[7] Agocs T. et al, "Final optical design for the WEAVE two-degree prime focus corrector", Proc. SPIE 9147-266 (2014)

[8] Terret D. L. et al, "Fibre positioning algorithms for the WEAVE spectrograph", Proc. SPIE 9152-23 (2014)

[9] Sayède F. et al, "WEAVE MOS Fibre Bundle Test Plan", Proc. SPIE 9151-227 (2014)

[10] Craig B. Markwardt, https://www.physics.wisc.edu/ craigm/idl/ 